\title{
Detection and Parameter Estimation of Polyphase Codes Radar Signal Based on Fractional Autocorrelation
}

\author{
WANG Xiao-feng \\ Dept. Electronic Engineering of \\ Naval Aeronautical and Astronautical University \\ Yantai, China \\ wxf870516@126.com
}

\author{
ZHANG Guo-yi, Zhou Rui \\ Dept. Electronic Countermeasure of \\ Aviation University of Air Force \\ Changchun, China
}

\begin{abstract}
Polyphase codes radar signals (Frank, P1, P2, P3and P4 codes) derived from linear frequency modulated (LFM) waveforms have been widely used in low probability of intercept (LPI) radar. To solve the problem of higher computational complexity in detection and parameter estimation of the polyphase codes radar signals, a fractional autocorrelation based algorithm is proposed. The paper proved that the fractional autocorrelation at an arbitrary angle exactly corresponds to the radial slice of the signal ambiguity function (AF) at that particular angle, based on which a detection method of polyphase codes radar signal is proposed. After that the parameter of the polyphase codes radar signal was estimated via the combination of fractional autocorrelation and fractional Fourier transform (FrFT). The proposed method, which does not compute the timefrequency plane and can be achieved by the FFT, observably reduces the computational complexity of polyphase codes radar signal detection and parameter estimation. The computer simulations verify the effectiveness of the proposed method.
\end{abstract}

Keywords - component; Signal detection; Parameter estimation; Polyphase codes radar signal; Fractional autocorrelation; Fractional Fourier transform

\section{INTRODUCTION}

To combat the increasingly powerful electronic countermeasure systems, more and more radar adopt the pulse compression signals which can obtain excellent performance of low probability of intercept (LPI). Polyphase codes radar signal (Frank, P1, P2, P3, and P4 codes), which derived linear frequency modulated (LFM) signal, is a commonly signal LPI signal because of its easy digital implementation, its versatility, and its high range resolution and Doppler tolerance $[1,2]$. The application of the polyphase codes radar signal in LPI radar system has brought a great challenge to the electronic intelligence (ELINT) systems. Thus, the detection and parameter estimation of polyphase codes radar signals has attracted much attention.

Polyphase codes radar signals has a similar time-frequency distribution characteristics with LFM signal. Thus, the method of signal detection and parameter estimation with LFM signal can be used to process polyphase codes radar signals. In [3], the Wigner-Ville distribution (WVD) characteristics of polyphase codes radar signal was analyzed. The researches proposed a polyphase codes radar signal detection and parameter estimation algorithm base on the Radon-Wigner transform (RWT) [4]. In low SNR the good detection and parameter estimation performance of RWT was specifically discussed in [5]. Due to the calculation of the time-frequency distribution and the two-dimensional search of Radon transform, the RWT method has huge computational cost. In [6-8], the authors proposed a polyphase codes radar signal detection method based on the Radon-Ambiguity transform (RAT). The RAT method reduced the two-dimensional search in RWT to one-dimensional search utilizing the characteristics that the ambiguity function of polyphase codes radar signal through the origin. However, the RAT method cannot estimate carrier frequency the signal. In [9], the integral quadratic phase function (IQPF) is applied to detection the polyphase codes radar signal. The IQPF method achieves polyphase codes radar signal detection and parameter estimation by accumulating the energy of quadratic phase function. All the above methods can effectively accomplish the detection and parameter estimation of polyphase codes radar signal, but they all have the problem of high computational complexity. The RWT and RAT both require the calculation of time-frequency matrix which is highly complicated, and then accomplish the detection and parameter estimation by Radon transform. Although the IQPF not require Radon transform, but it actually also calculates the time-frequency matrix.

To solve the problem of high computational complexity in the detection and parameter estimation of polyphase codes radar signal, this paper proved the equivalence relation of ambiguity function radial slice and fractional autocorrelation in theory, based on which a novel detection and parameter estimation method is also proposed. We detect the polyphase codes radar signal and estimate its frequency modulation slope base on the characteristics of fractional autocorrelation, and estimate the other modulation parameter of polyphase codes radar signal using the FrFT. The computational complexity and detection performance of the proposed method was discussed. At last the simulation experiments have verified the effectiveness of the proposed method. 


\section{THE POLYPHASE CODES RADAR SIGNAL}

Polyphase codes radar signals can be expressed analytically as follow

$$
x(t)=A \exp \left[j\left(2 \pi f_{c} t+\phi_{k}\right)\right], \quad 0 \leq t \leq T
$$

Where $A$ is the signal amplitude, $f_{c}$ is the carrier frequency, $T$ is the coding cycle, $\phi_{k}$ is the phase modulation function. Different phase modulation function represents different polyphase codes radar signal. Among them, Frank, P1, and P2 code is an approximation to LFM, using frequency step of number $L$, and sampling $L$ discrete phase for each frequency. The P3 and P4 code is derived from the sampling to LFM signal with Nyquist sampling rate [2].

Let $t_{b}$ is the code width of signal, $N_{c}$ is the code sequence length, $B$ is the signal bandwidth. The modulation parameters of the polyphase codes radar signal have the relationship as follow

$$
\begin{aligned}
t_{b} & =1 / B \\
T & =N_{c} t_{b}
\end{aligned}
$$

Normalized frequency modulation slope of polyphase codes radar signal is

$$
m=2 \pi / N_{c} t_{b}^{2}
$$

\section{FRACTIONAL AUTOCORRELATION}

The fractional autocorrelation of signal can be defined as

$$
R_{\phi}(\rho)=e^{j \pi \rho^{2} \cos \phi \sin \phi} \int s(t) s^{*}(t-\rho \cos \phi) e^{-j 2 \pi t \rho \sin \phi} d t
$$

Where $\phi$ is the fractional rotation angle. For $\phi=0$ and $\phi=\pi / 2$, signal fractional autocorrelation simplifies to the traditional time domain autocorrelation and frequency domain autocorrelation

$$
\begin{gathered}
R_{0}(\rho)=\int s(t) s^{*}(t-\rho) d t \\
R_{\pi / 2}(\rho)=\int|s(t)|^{2} e^{-j 2 \pi t \rho} d t=\int S(f) S^{*}(f-\rho) d t
\end{gathered}
$$

Where $S(f)$ is the Fourier transform of the signal $s(t)$. If $0<\varphi<\pi / 2$, fractional autocorrelation is something between time domain autocorrelation and frequency domain autocorrelation.

The fast algorithm of fractional autocorrelation was obtained using the fractional operators' theory in [11]

$$
R_{\phi}(\rho)=\mathrm{FFT}^{-1}\left\{\left|S^{\phi+\pi / 2}(u)\right|^{2}\right\}
$$

Where $S^{\varphi+\pi / 2}(u)$ represents the FrFT whose rotation angle is $\phi+\pi / 2$. As is shown in equation (8), the fractional autocorrelation can be implemented by one time FrFT and one time inverse FFT. Its computational complexity is very low.

The ambiguity function of signal can be defined as

$$
A F(\tau, v)=\int_{-\infty}^{+\infty} s(t+\tau / 2) s^{*}(t-\tau / 2) e^{-j 2 \pi v t} d t
$$

Where $\tau$ represents the delay, $v$ represents Doppler frequency shift. Ambiguity function is a powerful tool for analyzing polyphase codes radar signal. The ambiguity function of polyphase codes radar signal has the characteristics of multiple parallel ridges, and the main ridge which has maximum energy is certain to pass the origin coordinate. The signal detection and parameter estimation can be accomplished by extracting the ridges in the ambiguity function plane. Fig.1 displays the ambiguity function of P4 codes signal, other polyphase codes radar signal has a similar ambiguity function characteristics to it.

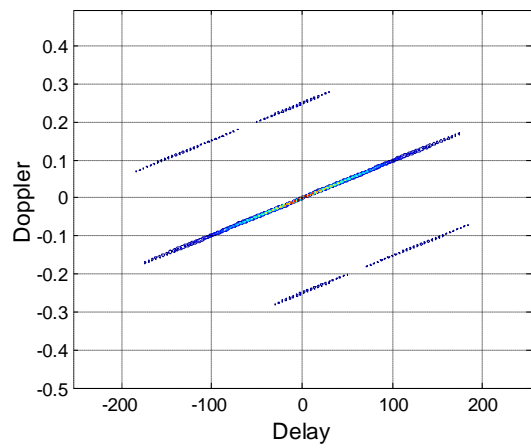

Fig. 1 P4 code ambiguity function

The relationship between the fractional autocorrelation and ambiguity function are discussed as below. Change the integration dummy variable $t=t^{\prime}-\frac{\rho \cos \varphi}{2}$ in (5) to obtain

$$
R_{\phi}(\rho)=\int s\left(t^{\prime}+\frac{\rho \cos \phi}{2}\right) s^{*}\left(t^{\prime}-\frac{\rho \cos \phi}{2}\right) e^{-j 2 \pi t^{\prime} \rho \sin \phi} d t
$$

Comparing (10) with (5), fractional autocorrelation and the ambiguity function have the relationship as follow

$$
\begin{aligned}
R_{\phi}(\rho) & =A F(\rho \cos \phi, \rho \sin \phi) \\
& =\left.\frac{1}{\cos \phi} A F(\tau, m \tau)\right|_{m=\tan \phi}
\end{aligned}
$$

Equation (11) indicates that the fractional autocorrelation is equivalent to the radial slice of ambiguity function. That is to say, fractional autocorrelation with the rotation angle $\phi$ equals the ambiguity function radial slice which is along the line $v=\tau \tan \phi$. Thus, the fractional autocorrelation can be used to process the polyphase codes radar signal as the ambiguity function. The relationship between the fractional autocorrelation and ambiguity function is shown in Fig.2.

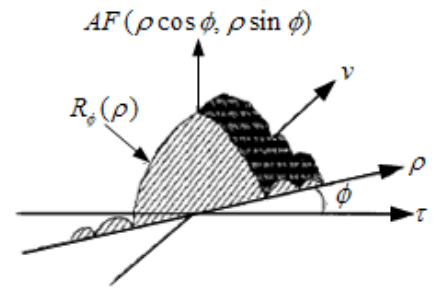

Fig.2 Fractional autocorrelation and ambiguity function

\section{Signal Detection AND PARAMETER Estimation}

RAT is an effective method to process polyphase code radar signal. The RAT of signal is the integration along the line $v=m \tau$ in the ambiguity function plane

$$
R A T(\phi)=\left.\int_{-\infty}^{+\infty}|A F(\tau, m \tau)| d t\right|_{m=\tan \phi}
$$


Where, $\phi$ is the rotation angle of the Radon transform, $m$ is frequency modulation slope of signal. The signal detection and frequency modulation slope estimation can be accomplished by searching the peak of RAT. The equivalence relation of ambiguity function radial slice and fractional autocorrelation has been proved. As is shown in (11) and (12), the fractional autocorrelation of signal is equivalent to the RAT of corresponding rotation angle. Thus, the integration fractional autocorrelation (IFA) which is the detection statistic basing on the fractional autocorrelation is defined as follow

$$
\operatorname{IFA}(\phi)=\int_{-\infty}^{+\infty} \mid R_{\phi}(\rho) d \rho
$$

If the peak value of detection statistic exceeds the detection threshold, the detection is successful; otherwise the polyphase codes radar signal does not exist. After successfully detecting the polyphase code radar signal, the frequency modulation slope can be estimated by the position of the peak value

$$
\hat{m}=\arctan (\hat{\phi}), \hat{\phi}=\arg \max \{\operatorname{IFA}(\phi)\}
$$

After completing the detection and modulation frequency slope estimation, the carrier frequency, bandwidth, and coding cycle can be estimated by calculating the FrFT in the fractional rotation angle corresponding to frequency modulation slope. The FrFT corresponding to the angle of frequency modulation slope is shown in Fig.3. The estimation method can be expressed as follow

$$
\begin{aligned}
f_{0} & =u_{0} \csc \hat{\phi} \\
B & =d \sec \hat{\phi} \\
T & =d \csc \hat{\phi}
\end{aligned}
$$

Where $u_{0}$ is frequency corresponding to maximum value of

FrFT, $d$ is the interval distance between the peak value in FrFT. The code with and the code sequence length can be calculated by the (2) and (3).

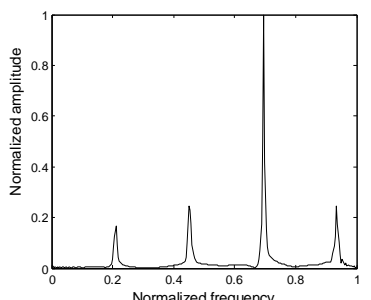

(a) $\mathrm{P} 1, \mathrm{P} 2$, and $\mathrm{P} 4$ code

Fig.3 FrFT at angle of frequency modulation slope.

\section{Performance Analysis and Simulation}

\section{A. Algorithm complexity analysis}

Let $N$ is the signal length, $M$ is the discrete number of searching angle. The algorithm complexity based on RAT and IFA will be analyzed as follow.

The complexity of calculating the ambiguity function plane is $O\left(N^{2} \log _{2} N\right)$. The complexity of implementing one time Radon transform is $O(N)$. Thus, the complexity of whole signal detection and parameter estimation algorithm based on the RAT is $O\left(N^{2} \log _{2} N+M N\right)$.
The complexity of using FFT to calculate one time discrete FrFT is $O\left(N \log _{2} N\right)$ the complexity of inverse FFT is also $O\left(N \log _{2} N\right)$. Thus, the complexity of implementing one time discrete fractional autocorrelation is $O\left(2 N \log _{2} N\right)$. If we want to calculate the fractional autocorrelation for $M$ different angle, then the total cost is $O\left(2 M N \log _{2} N+M N\right)$. The computational complexity of the following fixed angle FrFT is very small and can be negligible.

Because the RAT method needs to calculate the twodimensional plane of ambiguity function and detects the linear feature using the Radon transform, there is an exponential increase with the number of data points. While the IFA method needn't to calculate two-dimensional plane, and can be implemented by FFT, which is fit in with engineering application. Actually, the approximate range of signal frequency modulation slope is known, yet the RAT method still calculates the whole ambiguity function plane which is extremely complicated. The IFA method can calculates the detection statistics in a given range, which is more suitable for practical applications.

\section{B. Detection performance analysis and simulation}

As the equivalence relation of detection method based on ambiguity function and fractional autocorrelation, the detection performance of RAT was firstly analyzed before discussing the detection performance of IFA. The detection performance of RAT is related to the main ridge energy of ambiguity function. The larger the main ridge energy, the better performance signal detection. So the detection performance of different code type is different. In [6], the ratio relationship of ridge energy between LFM signal and polyphase code radar signal is given by simulation. Tab. 2 displays the ratio relationship.

\begin{tabular}{cccccccc}
\multicolumn{7}{c}{ Tab.2 } & \multicolumn{7}{l}{ The ratio relationship of ridge energy } \\
\hline Type & LFM & Frank & P1 & P2 & P3 & P4 \\
\hline Main ridge energy & $E$ & $0.44 E$ & $0.72 E$ & $0.73 E$ & $0.44 E$ & $0.77 E$ \\
\hline The complexity & of & the polyphase & codes & radar & signals
\end{tabular}
prevents an analytical analysis to the relationship of false alarm probability, detection probability, and SNR. To evaluate the effectiveness of the proposed detection statistics, we apply it to the simulated polyphase codes radar signals. The detection performance of the IFA is shown using the receiver operating characteristic (ROC). Simulation parameters are as follows. The signal carrier frequency is $f_{c}=10 \mathrm{MHz}$, the sampling frequency is $f_{s}=70 \mathrm{MHz}$, the code width is $t_{b}=0.1 \mu \mathrm{s}$, the coding cycle is $T=6.4 \mu \mathrm{s}$, the frequency modulation slope of LFM is $m=2 \pi / N_{c} t_{b}^{2}$. Fig. 4 is the ROC as probability of false alarm is $10^{-2}$.

As is shown in Fig.4, there is different detection performance for different code type of polyphase codes radar signal, and the detection performance of any polyphase codes radar signal is inferior to the LFM signal. Among the polyphase code radar signal, the P4 code signal has the best detection performance, the follow are P1, P2, P3, and Frank code. The detection performance of different code type is consistent with the main ridge energy in Tab.2. 


\section{Parameter estimation performance}

In order to verify the parameter estimation performance of the proposed method, five kinds of polyphase codes radar signal was simulated, respectively. In the simulation experiment, we ran the simulations 1000 times for each SNR. Fig.5 is the root mean square error (RMSE) in each SNR. The RMSE of parameter estimation is defined as

$$
R M S E=\frac{1}{N_{S}}\left(\sum_{n=1}^{N_{S}}\left(\zeta_{n}-\zeta^{\prime}\right)^{2}\right)^{1 / 2}
$$

Where $N_{S}$ is the number of simulations, $\zeta^{\prime}$ is the true value of the parameter, $\zeta_{n}$ is parameter estimation value in the $n$th experiment.

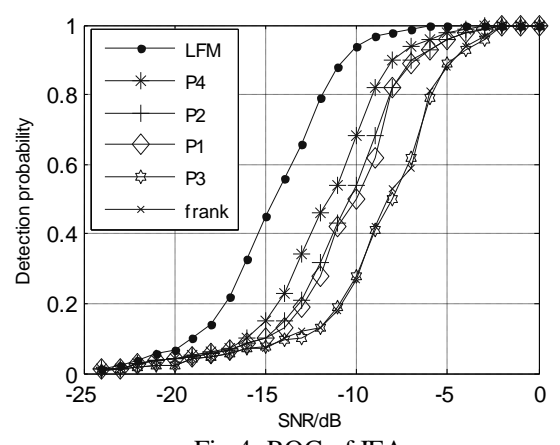

Fig.4 ROC of IFA

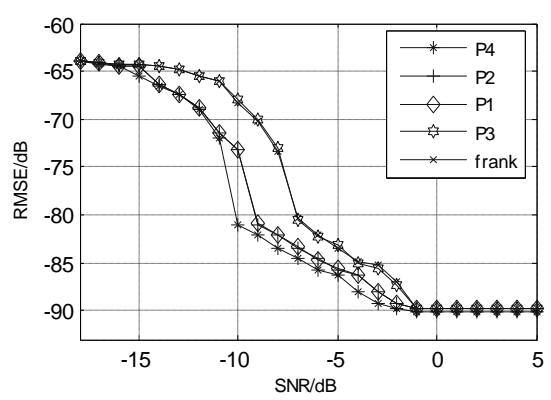

(a) frequency modulation slope.

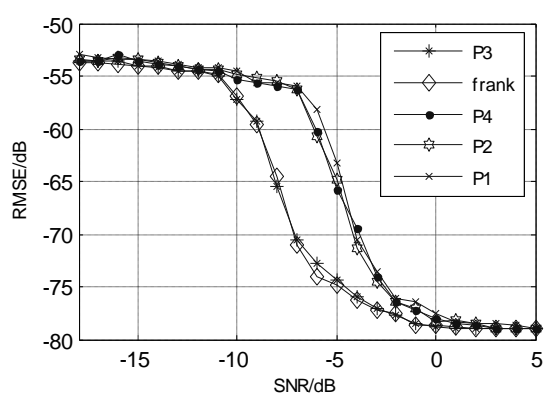

(b) code width

Fig.5 RMSE of parameter estimation.

As is shown in Fig.5(a), the SNR threshold of the frequency modulation slope estimation is ranked as follow: P4 code is about $-10 \mathrm{~dB}, \mathrm{P} 1$ and $\mathrm{P} 2$ code are about $-9 \mathrm{~dB}$ which is slightly higher than $\mathrm{P} 4$ code, $\mathrm{P} 3$ and frank code are about $-7 \mathrm{~dB}$ which is higher than others. The SNR threshold rank of code width estimation is shown in Fig.5(b): P3 and frank code have a lower SNR threshold than P1, P2 and P4 code. The reason for this phenomenon is still related to the ridge energy distribution of polyphase codes radar signal. As the P1, P2 and $\mathrm{P} 4$ code have a main ridge which has larger energy, the SNR threshold of the frequency modulation slope is lower than the P3 and frank code. But the P3 and frank code have a second ridge which has larger energy, therefore they have a lower SNR threshold than P1, P2 and P4 code in the estimation of code width. In addition, after reaching certain SNR threshold, the accuracy of parameter estimation will not be increased, this is result of the resolution.

\section{CONCLUSION}

The fractional autocorrelation characteristics of polyphase codes radar signal was discussed in this paper. After proving the equivalent relationship between the fractional autocorrelation and the radial slice of ambiguity function, a polyphase codes radar signal detection and parameter estimation method is proposed. The proposed method, which can be implemented by FFT, has solved the problem of high computational complexity in traditional method. The proposed method provides a new idea for processing polyphase codes radar signal, and has a certain engineering application value.

\section{REFERENCES}

[1] Pace P E. Detecting and classifying low probability of intercept $\operatorname{radar}[\mathrm{M}]$. Norwood, MA: Artech House, 2004: 133-165.

[2] XU Hui-fa, HU Xiao-feng, ZHANG Ming-zhi. Detection and parameter estimation of polyphase code radar signals based on Fractional Fourier transform[J]. Acta Armamentarii, 2012, 33(9): 1048-1054.

[3] Milne P R, Pace P E. Wigner distribution detection and analysis of FMCW and P-4 polyphase LPI waveforms[C]//Proc. IEEE International Conference on Acoustics, Speech, and Signal Processing, 2002: 39443947.

[4] Gulum T O, Pace P E, Cristi R. Extraction of polyphase radar modulation parameters using a Wigner-Ville distribution-Radon transform[C]// Proc. of the IEEE International Conference on Acoustics, Speech and Signal Processing, 2008: 1505-1508.

[5] XU Hai-yuan, ZHOU Yi-yu, HUANG Zhi-tao. Detection and parameter estimation method for Frank code pulse compression waveforms[J]. Systems Engineering and Electronics, 2007, 29(6): 858-861.

[6] Jennison B K. Detection of polyphase pulse compression waveforms using the Radon-Ambiguity transform $[\mathrm{J}]$. IEEE Trans. On Aerospace and Electronic Systems, 2003, 39(1): 335-343.

[7] XU Hai-yuan, HUANG Zhi-tao, ZHOU Yi-yu. Parameter estimation methods for polyphase codes derived from LFM waveforms using the Radon transform[J]. Signal Processing, 2008, 24 (2) :172-176.

[8] LI Hong, QIN Yu-liang, LI Yan-peng. Parameter estimation of intra-pulse modulation for poly-phase coded pulse based on $\mathrm{AF}[\mathrm{J}]$. Systems Engineering and Electronics, 2010, 32(9): 1823-1827.

[9] DU Qing, WANG Jian. Research of detection and parameter estimation for P3/P4 polyphase codes signal[J]. Science Technology and Engineering, 2013, (19): 5673-5678

[10] Olcay Akay, D. Faye Boudreaux-Bartels. Fractional convolution and correlation via operator methods and an application to detection of linear FM signals[J]. IEEE Trans. on Signal Processing, 2001, 49(5): 979-993.

[11] Olcay Akay, Erten Erözden Employing fractional autocorrelation for fast detection and sweep rate estimation of pulse compression radar waveforms[J]. Signal Processing, 2009, 89(12): 2479-2489.

[12] LI Jian, ZHANG Guo-yi, ZHANG Xu-zhou. Parameter estimation method for poly-phase coded signal based on the modified FrFT[J]. Modern Defence Technology, 2013, 41(4): 88-93. 